DOI: $10.17725 /$ rensit.2019.11.307

\title{
Reduction of graphene oxide by supercritical isopropanol: definition of acetone in the composition of the multicomponent mixture by gc-ms method
}

ElenaV. Fatyushina, ElenaYu. Buslaeva

Kurnakov Institute of General and Inorganic Chemistry. Russian Academy of Sciences, htto://www. igic.ras.ru/ Moscow 119991, RussianFederation

E-mail:fat@igic.ras.ru,buslaeva@igic.ras.ru

Received 20.08.2019, peer reviewed 20.09.2019, accepted 23.09.2019

Abstract. The analytical procedure of the reduction products of inorganic oxides and graphene oxide by supercritical isopropanol (propanol 2) has been developed. The method is based on the analytical control of acetone yield, which is always formed as a result of supercritical isopropanol reduction of these compounds. The presence of acetone is a confirmation of the reduction processes found by other methods. The problem of acetone identification, eluating as one peak with propanol 2 , which is present in a high concentration in a multicomponent mixture, is the "pass" of the substance in the sample under standard analysis conditions.

Keywords: graphene oxide, gas chromatography-mass spectrometry, selective detection, isomers, quantitative analysis of acetone

UDC 541.64.543.422.4

For citation: ElenaV. Fatyushina, ElenaYu. Buslaeva. Reduction of graphene oxide by supercritical isopropanol: definition of acetone in the composition of the multicomponent mixture by gc-ms method. RENSIT, 2019, 11(3):307-314. DOI: 10.17725/rensit.2019.11.307.

\section{Contents}

1. INTRODUCTION (307)

2. Materials and methods (308)

3. Results AND discussions (309)

4. Conclusion (313)

\section{ReferenCes (314)}

\section{INTRODUCTION}

In some cases, when there is no direct evidence of the processes of transformation of the substance in the solid phase, or it is difficult for a number of reasons (for example, an insufficient amount of the sample for the study by RFA), the presence of indirect evidenceacetone, which is always formed as a result of the interaction of supercritical alcohols and inorganic compounds-is indispensable. In other cases, the presence of acetone is a confirmation of the recovery processes detected by other methods. The presence of complex salts and mixtures of components in graphene oxide reduction products imposes additional requirements for the selectivity of acetone determination, which is satisfied by the gas chromatography-mass spectrometry (GCMS) method and makes it difficult to identify it by chromatographic methods. It was found that the main factors leading to false negative results of HCMS analysis are the presence of background components eluting together with the target substances. The problem of identifying acetone eluting by a single peak with propanol 2 (isopropanol) present in a high concentration is the "pass" of the substance in the sample under standard analysis conditions.

The aim was to develop a method of analysis of the solutions of complicated composition, resulting from the interaction of supercritical alcohol 2 propanol and inorganic compounds, based on analytical control of the yield of acetone as a witness and criterion of recovery of oxides and complex salts (including graphene oxide), to find the optimal conditions of analytical control of the recovery processes, 
ensuring the separation selectivity of overlapping chromatographic peaks.

Propanol 2 with acetone is usually divided into polar columns with polyethylene glycol (PEG). Many polar phases have disadvantages (relative to mid-polar, low-polar and non-polar). Among such disadvantages is a relatively low tolerance to oxidants. This should be taken into account when developing a technique on columns with PEG or its derivatives, which include the fixed liquid phase: FFAP, DB-WAX, DB-FFAP. The service life of the PEG speaker is significantly shorter than that of the HP-5 speaker and does not exceed one year.

To determine the complex composition of a mixture of near-boiling components, it is recommended to use a column with a nonpolar phase (polydimethylsiloxane) [1]. In cases where this fails, it is necessary to resort to highresolution mass spectrometry, or to methods that "shift" the analytical peaks to a different region of mass numbers, for example, to other methods of ionization or to obtain derivatives for which the masses of the characteristic ions of the analyzed compounds have different values. Obviously, increasing the resolution leads to a decrease in signal intensity.

This complicates and increases the cost of the analysis procedure, in addition, it makes it difficult to obtain quantitative results.

In this paper, an attempt is made to develop a method for determining acetone in a near-boiling isopropyl alcohol without preliminary sample preparation and separation of components of a complex mixture, providing quantitative results and a low detection limit.

\section{MATERIALS AND METHODS}

The work was carried out on the chromatomass spectrometry system of the company "Agilent" (USA), consisting of a quadrupole mass analyzer Agilent 5973, gas chromatograph Agilent 6890. Chromatography was performed on capillary column HP- 5 with fixed phase $-5 \%$ diphenylylsiloxane and 95\% dimethylsiloxane, column dimensions $0.32 \mathrm{~mm} \times 50 \mathrm{~m} \times 0.52 \mu \mathrm{m}$.

The initial temperature of the column was $30^{\circ} \mathrm{C}$, the temperature programming speed $10^{\circ} \mathrm{C} / \mathrm{min}$, the final temperature of the column $-250^{\circ} \mathrm{C} / \mathrm{min}$.

Ionization of substances in the mass spectrometer was carried out in the mode of electronic shock, registration of chromatograms was carried out both on the total ion current and in the mode of measuring the current created by individual ions or their groups.

Conditions for registration of chromatograms and mass spectra:

- interface temperature $280^{\circ} \mathrm{C}$;

- detector temperature $150^{\circ} \mathrm{C}$;

- ionizing electron energy $70 \mathrm{eV}$;

- recorded mass interval 12-350 a. e. m.;

- the scanning speed of 4.27 scans/sec;

- the voltage at the multiplier $70 \mathrm{~V}$;

- resolution 0.5 a.e.m.;

- dosing of the sample was carried out with the help of "Hamilton" microsyringes with a volume of $10 \mu \mathrm{l}$, the volume of the dosed sample was $0.4 \mu l$.

Before the analysis of samples under the same conditions, blank samples of isopropyl alcohol containing no target component-acetone were analyzed.

During the evaporation of the sample, the "split" mode was used with the ratio of the amount of injected substance into the capillary column-discharge, 1:10, respectively.

The volumetric velocity of the carrier gas is $1 \mathrm{ml} / \mathrm{min}$.

Checking the mass Device SetTings and MONITORING THE STABILITY OF THE CALIBRATION CHARACTERISTIC

This procedure was carried out on perfluorotributylamine supplied to the device according to the instructions "Autotune" [2]. 
The molecular composition was determined by comparing the obtained mass spectra with the library mass spectra given in the MSD ChemStation data collection and processing program.

\section{Preparation of Calibration solutions of ACETONE IN PROPANOL}

Calibration solutions were prepared as follows: to 10 aliquot volumes $(1 \mathrm{ml}$ ) propanol 1 and propanol 2 were added 2, 5, 10, 20, 50, 100, 200 $\mu l$ of acetone solution, while its concentration made up $0.02 ; 0.06 ; 0.16 ; 0.25 ; 0.49 ; 0.98 ; 1.96$; $4.55 ; 8.3 ; 14.28 ; 20.0$ (\%vol.), respectively. An internal standard (propanol 1) was added to the acetone calibration solution in propanol 2 and subjected to GCMS analysis. The concentration of propanol 1 in the calibration mixture was $0.5 \%$ vol.

\section{RESULTS AND DISCUSSIONS}

It is established that the main factors leading to false negative results and the impossibility of visual identification are the presence of background components eluting together with the target substances. Fig. 1 the fragments
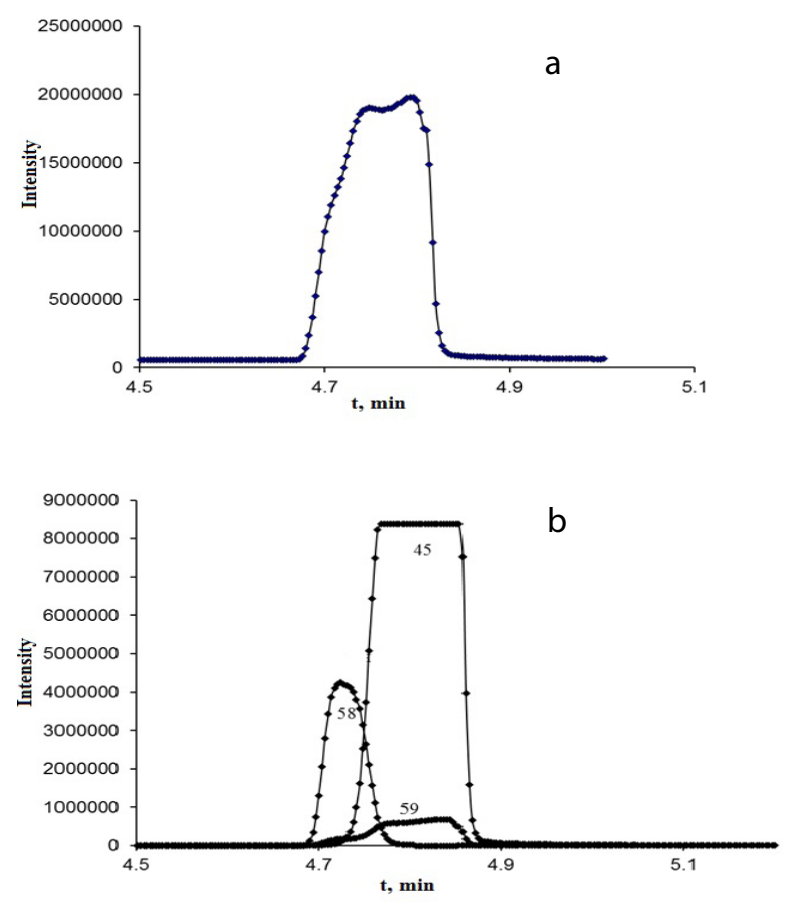

Fig. 1. The fragments of mass chromatograms of near-boiling compounds in the full-scan mass detection (a) and selected ion monitoring (SIM (b). of mass chromatograms of near-boiling compounds in the full-scan mass detection total ion current (a) and selected ion monitoring (SIM) in the extraction mode of characteristic ions $(b)$ are presented.

Due to these factors, especially at low acetone content in the solvent propanol 2, the method of determination, providing for the detection of the total ion current, was unsuitable. This fact is confirmed by a fragment of the chromatogram of the undivided components (a).

If the mass spectra of the undivided components have specific peaks characterizing each of the components and are absent (or insignificant) in the spectra of the other components, the ionic mass chromatograms for each of these characteristic peaks show the elution of each of their respective components regardless of whether the chromatograms $(b)$ overlap.

Determination of the acetone content in the matrix of near-boiling propanol 2 was carried out in the mode of ion chromatogram extraction and selective detection of characteristic lines 43 and $58 \mathrm{~m} / \mathrm{z}$ from the mass spectra without preliminary separation of these compounds during sample preparation.

A low-intensity peak $59 \mathrm{~m} / \mathrm{z}$ from the mass spectrum of propanol 2 was used as a label characterizing the change in chromatography and detection conditions to assess the true value of the acetone peak area. Additional information to control the stability of the measurement conditions was a constant ratio between the characteristic ions of the substance being determined.

Identification of acetone occurred by coincidence of maxima and character of curvature of peaks of ion chromatograms extracted from the total ion current. From Fig. $1 b$ it can be seen that it was possible to separate the chromatographic peaks and detect acetone.

The criterion of absence process reduction of oxides and complex salts was the absence of 
one chromatographic peak of the characteristic acetone ion within $0.05 \mathrm{~min}$ retention time in the presence of all other peaks.

In order to evaluate and eliminate interfering factors and obtain peaks of analytical ions free from superposition, isomers were used for calibration as analogues of the matrix component, but with different chromatographic characteristics, in this case: propanol 1. The physical properties of propanol 1 and propanol 2 are different: propanol 2 has a lower boiling point than propanol 1. It differs from its analogue retention time, the shift is about $2 \mathrm{~min}$. Chemical properties of these isomers, differing in the position of the functional group, differ slightly, because they have the same qualitative composition and nature of the bond between the atoms in the molecule. Fig. 2 illustrates the chromatographic peaks of acetone in propanol 2 (a) and in propanol 1 (b). It is seen that the peak of acetone overlaps with the peak of near-boiling propanol 2 and is independently measured in propanol 1.
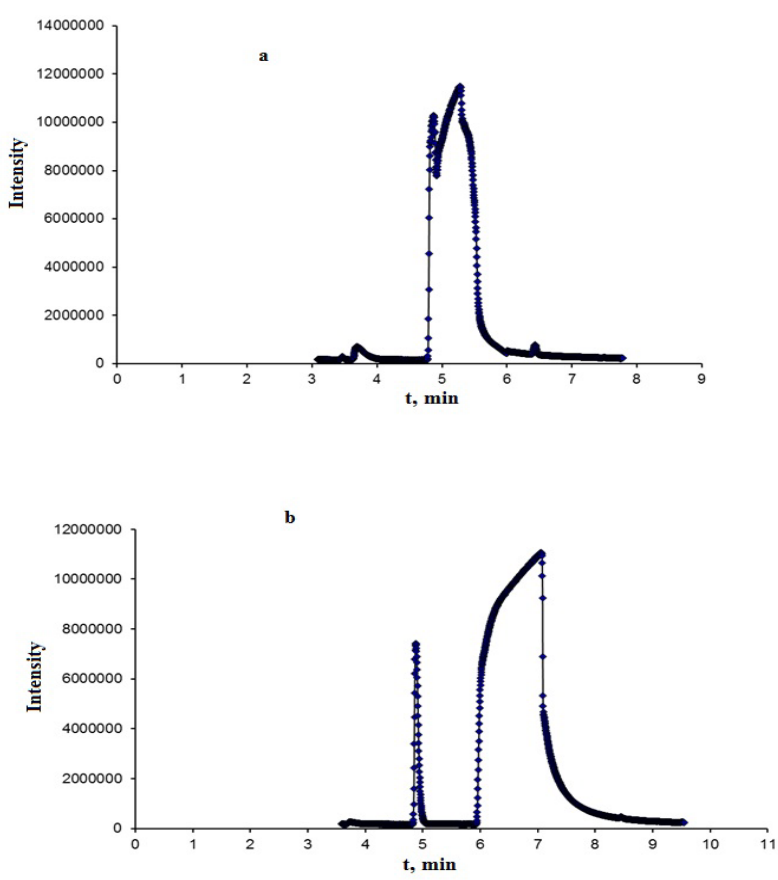

Fig. 2. Chromatographic peaks of acetone in propanol 2 (a) and in propanol 1 (b). It is seen that the peak of acetone overlaps with the peak of near-boiling propanol 2 and is independently measured in propanol 1.
Fig. 3 illustrates the difference between the mass spectra of propanol 2 and its isomer propanol 1, due to which separation by extractable ions is possible.

Mass chromatograms and mass spectra are given to illustrate the absence of the influence of solvent peaks of propanol 1 on the accuracy of the determination results (Fig. 2, 3) due to the absence of Isobaric interference.

\section{Quantitative GS MS analysis of aCETONE} IN A NEAR bOLING MULTICOMPONENT MIXTURE WITH ISOPROPANOL

Achieving high precision and the reproducibility of the quantitative results it is possible only with the right choice of quantitative analysis method and chromatographic analysis conditions. Quantification of identified compounds was performed by the method of "internal standard", for which a pre-determined calibration correction factor, indicating how many times the response of the mass-selective detector (area of the chromatographic peak is independent of the characteristic ion $\mathrm{m} / \mathrm{z} 58$ ) per unit mass of a substance is different from the response of the mass-selective detector per unit mass "internal standard" [3]. Its advantages are that it largely eliminates the impact of sample dosing error on the reproducibility of the analysis results.
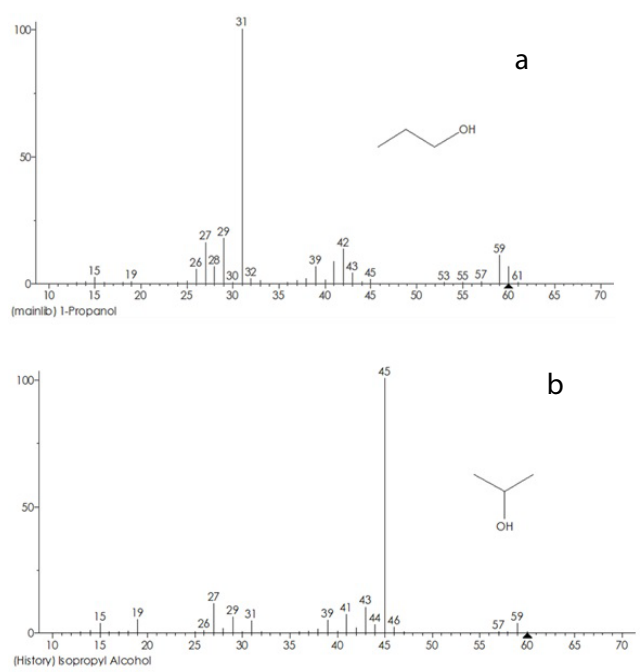

Fig. 3. Mass spectrum of propanol 1 (a) and propanol 2 (b). 


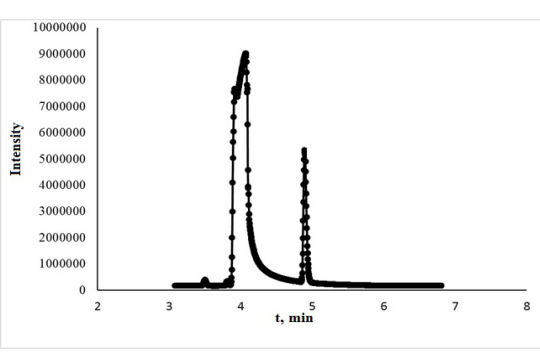

Fig. 4. Fragment of mass chromatogram of acetone in propanol 2 with internal standard propanol 1.

As an internal standard, propanol 1 was used, which has the same functional group with the matrix component propanol 2, but with different chromatographic and mass- spectral characteristics (Fig. 4).

The basis of quantitative chromatographic analysis dependence of peak height $b$ or its area $S$ on the amount of substance. Fig. 5 presented dependency relationship of the intensities of chromatographic peaks of acetone (corresponding to 58 ion) and propanol 1 in the propanol 2 matrix, calculated from the area and height of chromatographic peaks.

Using the $t$-criteria, it was found that the systematic error was significant against the background of a random spread in the calculation using the height of the peaks (Fig. 6), therefore, the quantitative assessment of the

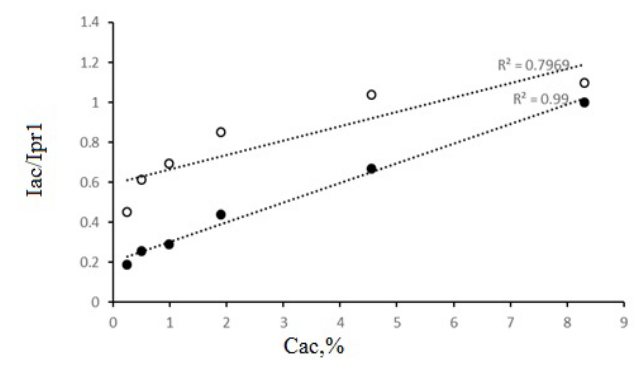

Fig. 5. Relations of analytical signals of acetone and internal standard of propanol $1\left(I_{a c} / I_{p r i}\right)$ depending on acetone content $\left(C_{a}, \%\right)$ calculated by area (๑) and by beights (口) of chromatographic peaks.

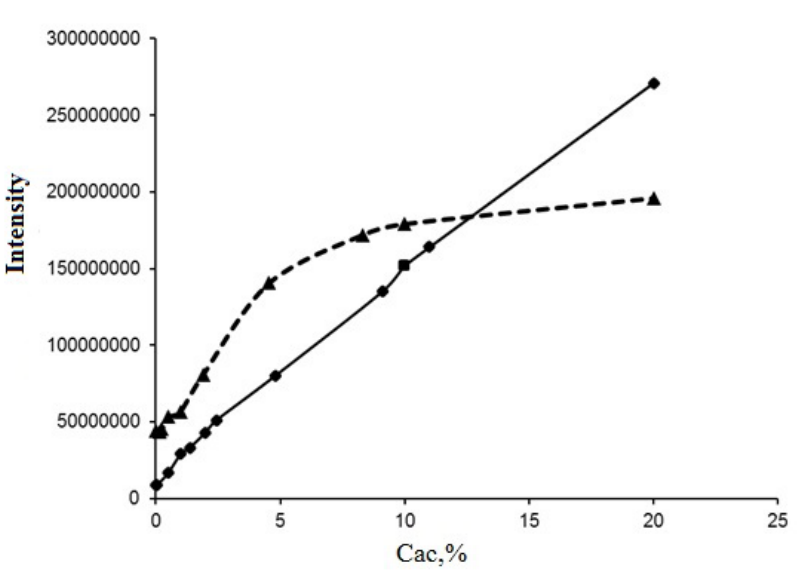

Fig. 6. Dependence of peak intensity on the amount of acetone analyzed in propanol 2 (-- -) and propanol 1 (-).

acetone content was carried out on the area of chromatographic peaks.

When working in the detector linearity range this dependence can be represented by the linear regression equation:

$C_{\mathrm{ac}}=K_{\mathrm{i}}^{*} C_{\mathrm{pr} 1} \times S_{\mathrm{ac}} / S_{\mathrm{pr} 1}$,

where $K_{i}$ - coefficient of relative sensitivity of acetone as the internal standard - propanol $1, C_{p r 1}-1$ propanol content in the mixture of sample and solvent (propanol 2), $S_{a c}$ and $S_{p r 1}$ - square peaks on the chromatogram, corresponding to ion $\mathrm{m} / \mathrm{z} 58$ acetone peak and the internal standard propanol 1. The value of $K$ does not depend on the amount of the determined component in the analyzed sample, in principle, should remain unchanged even when changing the conditions of chromatography and detection, unless, of course, these changes did not cause a violation of the linearity of the detector and the true value of the area was used as the peak parameter. This calculation scheme is widely used, but does not provide adequate results for nonlinear calibration dependencies. The use of calibration coefficients taken from the literature for quantitative calculations also leads to errors. In fact, it may be necessary to use combined versions of definitions or more complex modifications, in particular, the method of internal standard and absolute calibration [4]. 
QUANTITATIVE ANALYSIS OF SINGLE-PEAK ELUTING SUBSTANCES UNDER NONLINEAR DETECTION CONDITIONS

The main disadvantage of the quantitative analysis method proposed above is the need for non-overlapping characteristic ions to identify each compound, which is especially difficult to perform when analyzing compounds with similar structure, for which, as a rule, chromatographic and spectral overlap is most likely. A significant deviation of the calibration graph from the linearity can lead to the complete disappearance of small peaks.

Under these conditions, distortion of the shape and change in the area of the peaks were observed.

In order to expand the possibilities of quantitative analysis (the range of determined contents), it is additionally proposed to correct analytical signals using an external standard. To obtain peaks of analytical ions free from superposition, it is proposed to use isomersanalogues of the matrix component, having the same functional group, but with different chromatographic characteristics, in this case - propanol 1. The value of $K_{i}$ was determined by additional experiments involving a known amount of substance $i$.

On the example of a model mixture of acetone-propanol 1, acetone-propanol 2 (Fig. 6) the advantage of calibration using isomers is shown. Checking the isotope ratio revealed overlaps, which was expressed in a deviation from the linearity of the dependence of the intensity of the analytical signal of acetone on its content in propanol 2 (Fig. 6, dashed line). The most pronounced deviations in the calculation of height. This can be explained by the fact that the peak of acetone comes out just before the peak of propanol 2. This causes the acetone to enter the desorption zone, which gives its false peak at the same location. As a result, not only can the relative intensity of ion peaks in the mass spectra be distorted, but also excess peaks that are not characteristic of the analyzed substances may appear. The contribution to the intensity of peaks of characteristic background ions or peaks of ions of other components leads to overestimated results in determining the concentration of the analyte. It should be borne in mind that the amount of overlap can be large if it is due to the components present in the mixture in much larger quantities than those analyzed.

The distortion of the acetone peak shape was observed when the column was overloaded with a solvent eluting in a high concentration propanol 2.

The absence of interfering factors is illustrated by the linear dependence of the analytical signals of acetone on its content in propanol 1, including the imposition of a matrix "tail" is minimized (values on the $y$-axis are close to zero at " 0 "acetone concentration). In this case, the change in the analytical signals of acetone from its content in propanol 2 should be corrected beyond the linearity of the detector (Fig. 6). The effect of acetone concentration on the change in the ratio of acetone and propanol 1 peak areas is illustrated in Fig. 7.

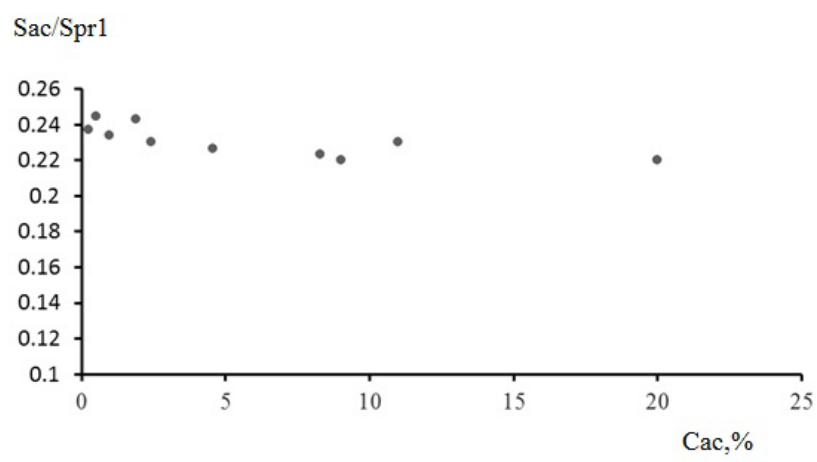

Fig. 7. Ratio of acetone and propanol 1 peak areas $\left(S_{a c} / S_{p r r}\right)$ depending on acetone concentration $\left(C_{a c} \%\right)$ in solvent. 
The correctness of the values of the calibration coefficient of acetone analytical signals corrected according to the external standard is confirmed by a small variation in the values of the relations between the areas of acetone peaks and the standard-propanol 1 present in equal concentrations.

If this condition is not met, the resulting errors can be compensated by using a combined version using an internal standard, wherein, in which the isomer of the matrix component is not an extraneous interfering component in the initial mixture. Uncontrolled changes in the absolute intensities of analytical signals are corrected relative to the internal standard or by the method of internal normalization to assess the relative amounts of components of mixtures [5]. The value of the ratio of the peak areas of the component and the introduced standard was determined and, using the previously obtained dependence of the ratio of the peak areas on the ratio of the quantities of these components in the model mixture, the content of the analyzed component was determined.

The amount of the $i$-th component $\left(Q_{i}\right)$ was calculated taking into account the known concentration of the standard $\left(C_{s t k}\right)$ in the calibration solution, the detector response to the internal standard $\left(S_{s t}\right)$ and the calibration dependence of the component-internal standard $\left(K_{s t}\right)$ :

$$
Q_{i}=K_{s t} C_{i k} * S_{s t} / C_{s t h}
$$

where $Q_{i}$ is the amount of the component equal to the product of its concentration on the injected volume, $C_{i k}$ - the concentration of the component in the calibration solution. The formula corrects the position of the calibration points along the component quantity axis, leaving the detector response value as it is obtained in the experiment.

The numerical values of the relative response coefficients of the detector are obtained from chromatographic data of calibration mixtures
Table

The method "introduced-found", mode SIM.

\begin{tabular}{|c|c|c|}
\hline $\begin{array}{c}\text { Detected } \\
\text { Content Range }\end{array}$ & $\begin{array}{c}\text { Introduced } \\
\mathrm{mg} / \mathrm{l}\end{array}$ & $\begin{array}{c}\text { Experimentally found } \\
\text { value }\end{array}$ \\
\hline $0.2-0.5$ & 0.3 & $0.35 \pm 0.07$ \\
\hline $0.5-1.5$ & 1.0 & $1.12 \pm 0.13$ \\
\hline $1.5-4.5$ & 2.8 & $3.04 \pm 0.26$ \\
\hline $4.5-20.0$ & 15.0 & $16.2 \pm 0.17$ \\
\hline
\end{tabular}

with known concentrations of standard and investigated impurities.

The estimation of the relative random component of the error of the results of quantitative determinations by the method of absolute calibration [6] was carried out according to the ratio:

$\delta_{\mathrm{Ci}}=\left(\delta_{\mathrm{ki}}+\delta_{\mathrm{Si}}\right) / 2$,

where $\delta_{k i}$ - the relative error of the $K_{i}$ coefficients, $\delta_{S i}$ - the same for the chromatographic peak areas of the substances to be determined.

The accuracy of the analysis was estimated by determining the boundaries in which the error of the results of the unit analysis is with a given probability $\mathrm{P}=0.95$.

The reproducibility of the introduction of samples was $1.5-2.5 \%$, the reproducibility of fixed retention times was not worse than \pm 0.05 min. The results of the determination of acetone in propanol 2 according to the developed method are presented in the Table.

\section{CONCLUSION}

The developed algorithm is useful for searching, identifying and determining the contents of overlapping a priori known components in a complex mixture (the qualitative composition is known) with a high degree of reliability.

The use of an isomer as an analogue of one of the undivided components for comparison made it possible to prevent overlaps of extracted ions, to increase isotopic sensitivity due to the absence of Isobaric interferences and to exclude the influence of the matrix effect on the analysis result. In addition, this eliminates the procedure of introducing a foreign component into the original mixture. 


\section{REFERENCES}

1. Berezkin VG, Lapin AB, Lipsky JB. Investigations of a new field in gas chromatography: Capillary columns with a super-thick layer of stationary liquid phase. $J$. Chromatogr.A, 2005, 1084(1-2):18-23.

1. Reese A, Prest H. Retention time locked GCMS analysis of phenols. Agilent Technologies application, 2001, 28(9):3934-5988.

2. Kai Meng C. Identification and Quantitation of Pesticides in the partsper-trillion range using retention time locking and GC/ MS. Agilent Technologies application, 2001, 14(11):4392-5988.

3. Kioussia MK, Angelisa YS, Cawleyc AT, Koupparisb M, Kazlauskasc R, Brennad JT, Georgakopoulosa CG. External calibration in GasChromatography-Combustion-Isotope Ratio Mass Spectrometry measurements of 92 endogenous androgenic anabolic steroids in sports doping control. J. Chromatogr. A, 2011, 1218:5675-5682.

4. Zenkevich IG, Kosman VM. Metody kolichestvennogo khromatograficheskogo analiza lekarstvennykh veshchestw [Methods of quantitative analysis of pharmaceutical substances]. S-P., SP KHFA Publ., 1999, 80 s.

5. Vial J., Jardy A. Quantitation by standard addition. Encyclopedia of Chromatography [Ed. J. Cazes], Taylor \& Francis, 2010, 3: 1975-1976. 